ÉGYPTE monde arabe

\section{Égypte/Monde arabe}

13 | 2015

Nouvelles luttes autour du genre en Egypte depuis 2011

\title{
Reconsidérer la dépolitisation : l'approche du témoin de HarassMap et la création d'une masse critique pour lutter contre le harcèlement sexuel en Égypte
}

Reconsidering de-politicization: HarassMap's bystander approach and creating

critical mass to combat sexual harassment in Egypt

\section{Angie Abdelmonem}

Traducteur : Mathilde du Pradel

\section{OpenEdition} Journals

\section{Édition électronique}

URL : https://journals.openedition.org/ema/3532

DOI : $10.4000 /$ ema.3532

ISSN : 2090-7273

Cet article est une traduction de :

Reconsidering de-politicization: HarassMap's bystander approach and creating critical mass to combat sexual harassment in Egypt - URL : https://journals.openedition.org/ema/3526 [en]

Éditeur

CEDEJ - Centre d'études et de documentation économiques juridiques et sociales

Édition imprimée

Date de publication : 10 novembre 2015

ISBN : 9782905838865

ISSN : 1110-5097

\section{Référence électronique}

Angie Abdelmonem, «Reconsidérer la dépolitisation : l'approche du témoin de HarassMap et la création d'une masse critique pour lutter contre le harcèlement sexuel en Égypte », Égypte/Monde arabe [En ligne], 13 | 2015, mis en ligne le 10 novembre 2015, consulté le 07 juillet 2022. URL : http:// journals.openedition.org/ema/3532; DOI : https://doi.org/10.4000/ema.3532 


\section{Reconsidérer la dépolitisation : l'approche du témoin de HarassMap et la création d'une masse critique pour lutter contre le harcèlement sexuel en Égypte}

Reconsidering de-politicization: HarassMap's bystander approach and creating critical mass to combat sexual harassment in Egypt

\section{Angie Abdelmonem}

Traduction : Mathilde du Pradel

\section{NOTE DE L'ÉDITEUR}

L'approche du témoin ou du spectateur, bystander approach en anglais, est une des approches liées à la prévention des violences sexuelles, qui encourage les individus à s'approprier le problème du harcèlement sexuel et à intervenir et s'exprimer lorsqu'ils sont témoins de harcèlement physique ou verbal. (Note du traducteur)

\section{NOTE DE L'AUTEUR}

Les informations sur lesquelles cet article est basé sont issues des recherches doctorales de l'auteure, pour lesquelles elle a bénéficié de la bourse $n^{\circ} 1357477$ de la National Science Foundation (USA).

Depuis le renversement de Hosni Moubarak en février 2011, le phénomène du harcèlement sexuel dans les lieux publics fait l'objet d'une attention grandissante. Dès 2008, le Centre Égyptien pour les Droits des Femmes (CEDF), une des premières ONG à 
faire spécifiquement campagne contre le harcèlement sexuel dans les lieux publics, publiait un rapport selon lequel $83 \%$ des Égyptiennes et $98 \%$ des étrangères enquêtées déclaraient avoir subi des actes de harcèlement sexuel ${ }^{1}$. La campagne du CEDF, "Rendre nos rues sûres pour tout le monde ", définissait le harcèlement sexuel comme un problème en grande partie culturel, social et psychologique et utilisait des techniques axées sur la communauté ${ }^{2}$ ainsi que des moyens technologiques novateurs afin d'accroître la sensibilisation de l'opinion publique à cette question ${ }^{3}$. Cependant, les détracteurs de cette campagne soutenaient que l'accent placé sur les causes sociales et culturelles du harcèlement sexuel ne conduisait qu'à des interventions sociales et culturelles, et non politiques ou économiques, dépolitisant ainsi le problème ${ }^{4}$. Les principaux aspects de cette critique reprochaient ainsi au CEDF le fait qu'il déconnectait le harcèlement sexuel quotidien de la violence sexuelle orchestrée par l'État en se concentrant sur le harcèlement de rue anonyme ciblant des " hommes dotés d'une mauvaise mentalité culturelle», et qu'il ne s'intéressait pas aux inégalités structurelles entre les sexes ${ }^{5}$.

2 Après la révolution, une nouvelle étude a été réalisée par UN Women ${ }^{6}$ en 2013. Celle-ci montre que le harcèlement sexuel dans les lieux publics, en particulier dans les rues et les transports, touche pratiquement toutes les femmes, $99 \%$ des enquêtées de l'échantillon ayant déclaré avoir été harcelées sexuellement. ${ }^{7}$ Presque la moitié de l'échantillon indique que le harcèlement sexuel a augmenté après la révolution, bien que la manière dont les répondantes parviennent à cette conclusion ne soit pas très claire. Il est probable que la révolution ait contribué à accroître la sensibilisation de l'opinion publique au harcèlement sexuel, entraînant le signalement d'un plus grand nombre de cas. De façon plus importante encore, la révolution a offert une occasion unique permettant l'essor de nombreuses initiatives communautaires de lutte contre le harcèlement sexuel ayant pour but le remodelage des normes sociales historiques et des comportements en matière de harcèlement sexuel. ${ }^{8}$ De telles initiatives ont fait le choix stratégique d'éviter les engagements politiques pour combattre le harcèlement.

3 S'intéressant aux arguments des détracteurs du militantisme anti-harcèlement sexuel, cet article vise à déterminer si les approches axées sur la communauté avant et après la révolution ont effectivement dépolitisé le problème du harcèlement sexuel. Il réfute les affirmations selon lesquelles les efforts de lutte contre le harcèlement du CEDF ont eu un effet de dépolitisation, et défend plus généralement l'idée que ces interventions visant à changer les normes et les comportements socioculturels sont plus politiques que leurs détracteurs ne veulent bien l'affirmer. Cette analyse est fondée sur la nécessité de reconsidérer ce qui est politique et le sens du concept de dépolitisation. Pour ce faire, elle souligne la "diversité des modalités de participation ayant le potentiel de mobiliser les registres moraux ordinaires ", c'est-à-dire les interventions centrées sur «l'ordinaire» qui redéfinissent la nature des pratiques et des responsabilités sociales et incitent les gens à des actions qui peuvent être politiques. Les détracteurs du militantisme anti-harcèlement se fondent sur une division particulière entre culture et politique, d'après laquelle les négociations sociales et culturelles sont rarement considérées comme des actes intrinsèquement politiques ${ }^{10}$. Parallèlement, ils ne considèrent pas la sphère politique comme «la cristallisation institutionnelle [...] de quelque chose qui se produit ailleurs, dans de multiples sites de contestation locaux, tels que le lieu de travail, les association et les institutions $»^{11}$. Cet article affirme que le militantisme communautaire concentré sur la redéfinition de 
normes culturelles relatives au harcèlement sexuel est un processus politique qui n'ignore pas les discriminations structurelles entre les sexes et dont les effets recherchés à long terme sont politiques et légaux.

\section{Le harceleur est un criminel}

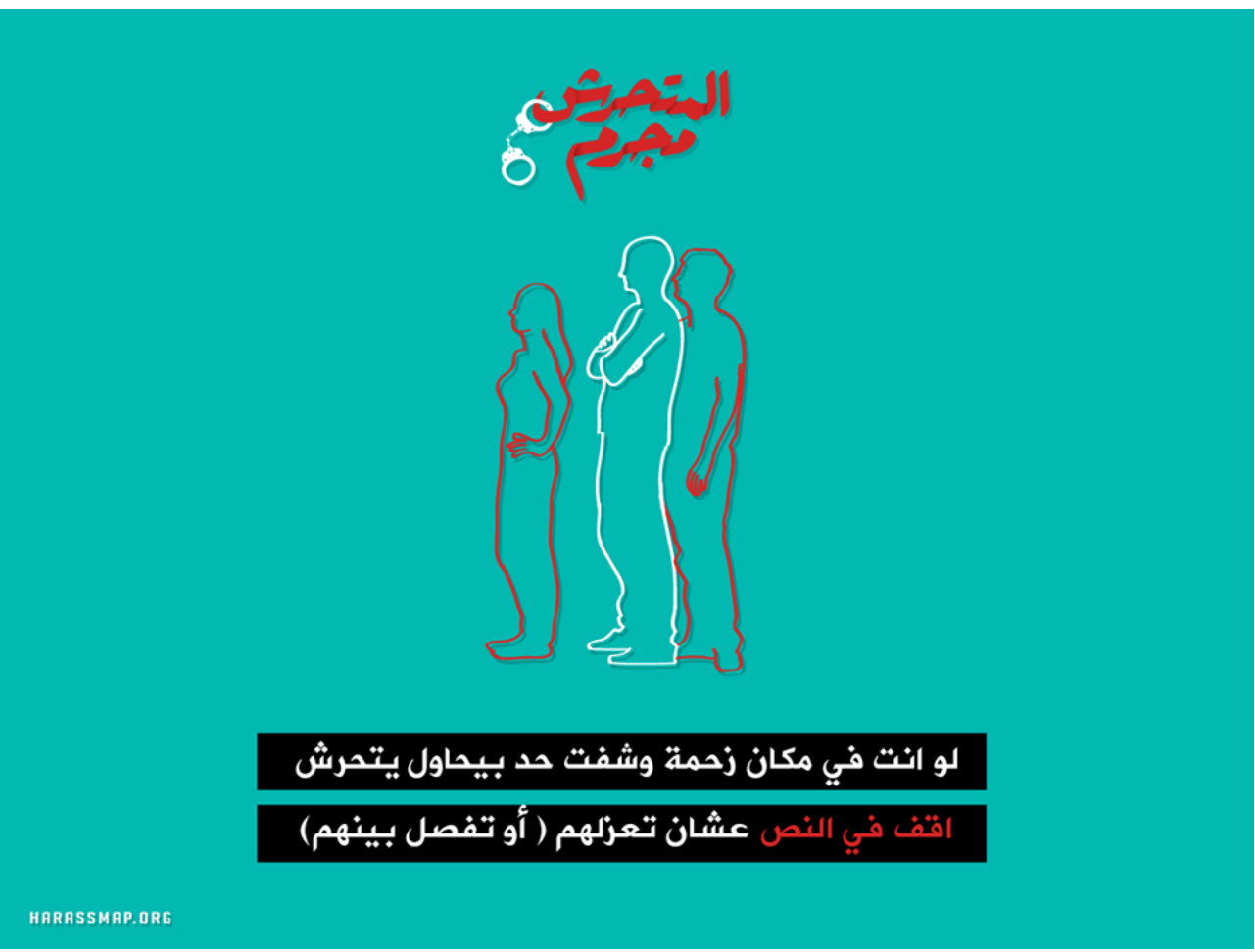

Cette image provident de la campagne "المتحرش مجرم" ("Le harceleur est un criminel ») menée par HarassMap en été 2015; c'est un exemple de leur travail de sensibilisation des spectateurs/témoins du harcèlement sexuel. La campagne a été conçue pour informer le public au sujet de la nouvelle loi criminalisant le harcèlement, mais aussi pour leur donner des conseils sur comment se rendre utiles en de telles situations sans nécessairement aller voir la police en premier lieu, ce qui peut être problématique.

4 L'initiative indépendante HarassMap est la principale étude de cas présentée dans cet article, étant donné le développement philosophique avancé de son approche stratégique. Par le biais d'un militantisme de rue et axé sur la communauté, cherchant à mobiliser des témoins, HarassMap fait usage de stratégies qui visent à remettre en cause et à remodeler les normes traditionnelles qui rendent le harcèlement sexuel socialement acceptable. Cela implique de bouleverser les normes de genre patriarcales (binaires), ainsi que d'encourager à la prise de responsabilité sociale au niveau intracommunautaire. Au travers de la négociation de nouvelles normes et de la construction d'un public socialement responsable, s'intéressant au bien-être des membres de la communauté et prêt à s'exprimer contre le harcèlement, HarassMap cherche à créer une masse critique qui finira par réclamer un changement politique et légal de la part des institutions étatiques. La discussion suivante prend pour point de départ les théories du changement des normes, le concept de public témoin et celui de masse critique, afin d'étudier la nature politique du militantisme communautaire de HarassMap, ainsi que la tentative de l'association d'agir sur le niveau d'engagement légal et étatique contre le harcèlement sexuel dans les lieux publics. Il convient cependant de noter ici que cet article ne traite pas la question de l'efficacité de l'action 
de HarassMap en la matière, mais seulement de la nature politique de cette tentative. Par ailleurs, des organismes tels que HarassMap n'ont pas de programme de changement explicite au sein de la sphère politique, mais défendent cependant le fait que le changement politique ne peut être durablement établi que si la population ellemême perçoit le harcèlement différemment.

\section{Les détracteurs du militantisme anti-harcèlement}

5 Tadros défend l'idée que le harcèlement en Égypte prend deux formes: l'une politiquement motivée, l'autre socialement motivée ${ }^{12}$. Le harcèlement sexuel socialement motivé relève du harcèlement de rue quotidien, qui prend généralement la forme de commentaires, de regards insistants, d'attouchements et d'autres gestes ${ }^{13}$. Le harcèlement politiquement motivé se produit en revanche largement dans le cadre de manifestations, et implique des groupes d'hommes harcelant les femmes. Selon beaucoup de militants, il est orchestré et financé par l'État dans le but d'évincer les femmes de l'espace public ${ }^{14}$. Pour Tadros, il était essentiel de faire apparaître cette distinction, afin d'éviter la confusion dans l'opinion publique et de faire la lumière sur le fait que l'État lui-même transgresse l'intégrité physique des femmes et se trouve fermement impliqué dans la définition sexiste de la notion de respectabilité ${ }^{15}$. Ces deux formes de harcèlement sont manifestes en Égypte depuis 2005, sinon avant. L'incident du Mercredi Noir de cette année-là est cité par les militants comme l'un des premiers cas de harcèlement sexuel, lors duquel des baltagiyya (voyous) ont été engagés par l'État égyptien pour harceler les femmes militantes contestant le référendum constitutionnel devant le syndicat des journalistes. Le cas de harcèlement de masse dans les rues du centre-ville du Caire lors des congés de l'Aïd en 2006 a mis en évidence l'importance croissante du problème du harcèlement sexuel ${ }^{16}$.

Durant cette période, le CEDF a constitué sa campagne pour définir et combattre le harcèlement sexuel dans les rues. Selon Rizzo et al. ${ }^{17}$, le CEDF était unique du fait de ses techniques communautaires créatives, comprenant notamment des journées de sensibilisation utilisant l'art et la musique, l'organisation d'activités bénévoles et la coopération avec le secteur des entreprises privées afin d'obtenir des services gratuits (pro bono) pour lutter contre le harcèlement sexuel. L'approche du CEDF insistait sur "l'importance de s'attaquer à une culture tolérant le harcèlement sexuel ${ }^{18}$. La campagne s'est pourtant vue reprocher de ne pas situer le harcèlement sexuel dans le système plus large de violence à caractère sexiste incluant la répression et la violence sexuelle perpétrées par l'État. ${ }^{19} \mathrm{Abu}$ Lughod affirmait ainsi que cette approche sociale et culturelle, ancrée dans les sphères du développement transnational et de l'entreprise capitaliste, contribuait à créer des femmes victimisées et des hommes culturellement mauvais. Pour Amar, au travers de leur fixation sur «la perversion libidinale des garçons de la classe ouvrière » et " les dangers de la masculinité », le CEDF s'est rendu complice de dépolitisation du harcèlement sexuel, en ne contestant pas l'usage par l'État de la violence sexuelle et de la torture et en réclamant au contraire un surcroit d'interventions du brutal État sécuritaire pour protéger les femmes dans les espaces publics. ${ }^{20}$ Cependant, le choix du CEDF de travailler au niveau communautaire a été fait dans un contexte de répression étatique contre la société civile, les ONG étant contraintes au silence, limitées et cooptées par l'État dans un climat corporatiste ${ }^{21}$. Les militants du CEDF investis dans la campagne anti-harcèlement sexuel s'interrogeaient 
sur l'efficacité d'une action de plaidoyer politique au sein d'une structure politique privant les ONG de leurs droits.

7 Entre 2010 et 2012, immédiatement avant et à la suite de la révolution de 2011, le nombre d'initiatives nées afin de lutter contre le harcèlement sexuel a augmenté. L'environnement politique corporatiste prérévolutionnaire, marqué par des fluctuations dans la répression étatique et l'inclusion de forces politiques d'opposition dans le processus politique, a été temporairement perturbé par l'instabilité politique ayant suivi la révolution. ${ }^{22}$ ElSayed et Rizzo affirment que le militantisme des femmes durant cette période était encore exclu du processus politique, leurs demandes pour plus d'égalité ignorées, et que les tensions entre forces laïques et religieuses concernant la nature des droits des femmes ont produit un ensemble distinct de contraintes politiques mais ont contribué à créer une opportunité unique pour les initiatives communautaires. Au cours des années de contestation, entre 2011 et 2014, le ciblage délibéré des femmes sur les lieux de manifestation est devenu évident. ${ }^{23}$ Les militants étaient alors véhéments dans leur condamnation de la conduite par l'État de tests de virginité sur des manifestantes, ainsi que dans leur condamnation d'acteurs étatiques suspectés d'engager des baltagiyya pour attaquer les femmes lors de manifestations, bien qu'il ait été depuis reconnu de façon nuancée que les agressions de masse et les viols à Tahrir aient été commis par un mélange de voyous embauchés par l'État et d'opportunistes. ${ }^{24}$ Dans ce contexte, plusieurs initiatives sont rapidement nées pour assister les victimes ou les survivantes ayant subi des violences lors de manifestations, parmi lesquelles Operation Anti-Sexual Harassment (OpAntiSH) et Tahrir Bodyguards. Composées d'une large base de militants bénévoles et de citoyens préoccupés par le problème, ces initiatives ont été de courte durée, ayant émergé en réponse à l'urgence de la situation des femmes place Tahrir. Il a ensuite été difficile pour ces deux initiatives d'adapter leur travail à des cadres de manifestation nonrévolutionnaires et à d'autres formes de militantisme, différentes de la seule manifestation.

8 D'autres initiatives indépendantes créées à cette époque, à l'image de HarassMap, du Mouvement Empreinte (Harakat Bassma, Imprint Movement) et du Mouvement antiharcèlement sexuel (Ded el-Taharush), ont concentré leurs efforts sur la modification des perceptions sociales et des comportements au sein des communautés plutôt que sur le lobbying auprès des responsables politiques. Parallèlement, un certain nombre d'ONG militantes ont intégré le harcèlement sexuel à leurs programmes de lutte contre la violence à l'égard des femmes. L'organisation Nazra pour les études féministes (Nazra for Feminist Studies) a par exemple commencé à promouvoir auprès des autorités étatiques l'établissement d'une stratégie nationale pour lutter contre toutes les formes de violence sexuelle, harcèlement sexuel inclus. ${ }^{25}$ Fatma Khafagy, la médiatrice du Conseil National pour les Femmes (National Council for Women) a cependant exprimé sa déception quant au fait que les militants anti-harcèlement continuaient à présenter largement le problème comme d'ordre culturel, en dehors du contexte politique, économique et sociologique dans lequel il est ancré. ${ }^{26}$ De son point de vue, mettre un terme au harcèlement social requiert une compréhension plus holistique et scientifique de ses différents aspects sous-jacents, qu'elle estime totalement absente du travail actuel des ONG et des initiatives anti-harcèlement. Tadros soulève également un problème concernant le fait que les interventions sociales centrées sur la «société » ou la "jeunesse", sans analyse politique concomitante, reproduisent des pratiques patriarcales, à l'image de la protection des femmes par le biais d'une limitation de leur 
accès à l'espace public. ${ }^{27}$ Des analyses récentes par Skalli notent cependant que les initiatives de jeunesse post-révolutionnaires ont été activement impliquées dans le bouleversement des normes de genre, ce qui suggère qu'elles n'ignorent en réalité pas les inégalités structurelles entre les sexes. ${ }^{28}$

Il ne fait pas de doute que la compréhension de la relation entre harcèlement sexuel de rue socialement motivé et violence sexuelle d'État politiquement motivée depuis la révolution est d'une importance cruciale. La révolution a été témoin d'une escalade de la violence, sinon du nombre réel de cas, en matière de harcèlement sexuel et d'agressions, en particulier dans le cadre de manifestations. Non seulement le gouvernement égyptien est suspecté d'avoir orchestré une grande partie des violences sexuelles collectives de Tahrir pour limiter la participation publique des femmes, mais l'État est aussi perçu comme le créateur d'une atmosphère d'impunité pour ceux qui pratiquent le harcèlement de façon quotidienne dans les rues, du fait de structures légales inadéquates et lourdes ainsi que de pratiques laxistes en matière d'application des lois. Néanmoins, une littérature de plus en plus abondante sur le sujet montre aussi que le harcèlement sexuel est une occurrence quotidienne à grande échelle fondée sur des normes différenciées selon le sexe et inégalitaires, perpétuées dans la vie de tous les jours. ${ }^{29}$ Selon Tadros, les agressions politiquement motivées et le harcèlement sexuel quotidien coexistent au sein du même système de pouvoir, de violence et de normes sociales qui tolèrent l'agression. ${ }^{30}$ Alors que le harcèlement politiquement motivé et le harcèlement socialement motivé peuvent être considérés comme deux aspects distincts du harcèlement sexuel, ils sont en fait similaires, se recoupent et sont deux expressions se renforçant mutuellement de normes sexistes discriminatoires qui désavantagent les femmes dans l'espace public. En tant que facettes de ce système de violence sexiste, les formes encouragées par l'État et les formes quotidiennes de harcèlement sexuel soulignent l'intégration des sphères socioculturelle et politique. Les interventions anti-harcèlement sexuel sont, par conséquent, exercées au sein de ce système unique, politiquement et socialement intégré de violence sexiste ; leur travail a une incidence à la fois dans le domaine social et dans le domaine politique.

\section{Normes, témoins et masse critique : considérations théoriques}

10 Finnemore et Sikkink définissent les normes « comme des critères de comportement appropriés pour des acteurs avec une identité donnée", soit des critères à la fois régulateurs et d'évaluation, englobant des comportements et des valeurs. ${ }^{31}$ Les théories du changement des normes sont souvent centrées sur le flux descendant, ou en cascade, des codes éthiques transnationaux vers les États et les populations locales, c'est-à-dire l'adoption de et l'adhésion à des conventions internationales et la codification de principes axés sur les droits dans les constitutions locales et les cadres législatifs..$^{32}$ La société civile joue un rôle significatif en ce qui concerne le processus d'internalisation ou de socialisation des normes. Les entrepreneurs de normes ${ }^{33}$ contribuent à créer, à encadrer et à diffuser de nouvelles normes en convaincant les gens de leur bien-fondé, et servent de groupes de pression travaillant en coordination avec d'autres organisations locales, régionales et internationales pour contraindre l'adhésion des États à ces normes. Ceci inclut le remodelage de normes problématiques au travers de pratiques d'encadrement qui peuvent s'appuyer sur les normes anciennes 
et enracinées, ou bien les contester directement, ou encore former un mélange hybride des deux. ${ }^{34}$ La négociation de nouvelles normes et la redéfinition de comportements sociaux et de valeurs est un processus intrinsèquement politique, étant donné que « les significations sont toujours constitutives de processus qui cherchent à redéfinir le pouvoir social ».35 Pourtant, c'est souvent l'institutionnalisation qui est perçue comme le moyen-clé par lequel les normes sont incorporées au contexte socio-politique.

11 Les militants anti-harcèlement sexuel travaillant au niveau communautaire mettent en doute la mesure dans laquelle les instruments politico-légaux seuls peuvent remodeler les normes sociales au sein de l'opinion publique et de la société. Le récit d'un cas de harcèlement sexuel dont a été témoin Hussein al-Shafe'i, directeur de l'unité d'intervention communautaire ${ }^{36}$ de HarassMap, constitue un exemple important de l'insuffisance du caractère dissuasif de la loi selon les militants: lors d'un trajet en minibus entre Alexandrie et le Caire, une femme a accusé de façon véhémente l'un des passagers assis derrière elle de la harceler sexuellement. Le minibus s'est arrêté alors que les passagers tentaient de calmer la femme, la pressant de laisser passer l'affaire et faisant valoir leur souhait de pouvoir rentrer chez eux. Celle-ci a demandé que l'homme soit forcé à descendre du véhicule, tandis que lui a affirmé haut et fort qu'il ne partirait pas. Le bus est reparti, l'homme étant toujours à bord. La femme a alors passé un coup de téléphone que tous les autres passagers ont pu entendre, insistant sur le fait que la police les attendrait à leur arrivée pour arrêter le harceleur. L'homme s'est soudain mis à crier en demandant au chauffeur de bus de le laisser sortir, hurlant que la femme "allait l'envoyer en enfer ». Les passagers ont tous été d'accord sur le fait qu'il valait mieux qu'il s'enfuie dans le désert plutôt qu'il soit arrêté. Pour al-Shafe 'i, au-delà du fait de ne montrer aucune préoccupation pour la femme harcelée, les passagers n'ont pas considéré le harcèlement sexuel comme un crime, et certainement pas comme un acte méritant une arrestation et des poursuites judiciaires. Malgré l'existence de lois interdisant les attentats à la pudeur, les militants de HarassMap affirment que les gens ne considèrent pas le harcèlement sexuel comme problématique, ou comme une infraction punissable. D'après el-Shafei, «les gens ne veulent mettre un terme au harcèlement, ils veulent simplement éviter de se faire prendre $»{ }^{37}$

Les efforts centrés sur le comportement des témoins, à l'image des passagers du bus dans l'exemple susmentionné, sont essentiels pour les initiatives de lutte contre le harcèlement. La recherche socio-psychologique a analysé de façon approfondie le phénomène communément connu sous le nom d'" effet du témoin ", c'est-à-dire le fait de se tenir à l'écart et de s'abstenir d'apporter une aide à une personne en détresse, lorsque d'autres personnes sont présentes. ${ }^{38}$ Il existe de nombreuses raisons permettant d'expliquer l'effet du témoin, dont l'inhibition, la peur de l'embarras, un processus de diffusion de la responsabilité qui se met en place au sein de la foule des témoins assistant à la situation, le coût élevé de l'intervention, le manque de compétences appropriées pour intervenir et le contrôle social exercé par la foule ellemême. ${ }^{39}$ Malgré cela, des recherches ont montré que des témoins peuvent s'exprimer lors qu'ils assistent à la transgression d'une norme s'ils se sentent personnellement concernés par celle-ci. ${ }^{40}$ La recherche sur la violence sexuelle a aussi souligné l'importance du comportement des témoins dans le changement des normes de la communauté en général. ${ }^{41}$ Par ailleurs, les militants sociaux peuvent s'inspirer des réactions des observateurs ou des témoins vigilants. Autrement dit, ces réactions peuvent guider l'intervention sociale. ${ }^{42}$ Le concept de «public témoin» est ici 
significatif. Ce public se compose d'individus qui n'adhèrent pas à un mouvement ou ne se sentent pas concernés par un problème, ou encore de "spectateurs distaux ", qui pourraient cependant apporter un témoignage et réagir à des problèmes liés à un mouvement social ou politique, et pourraient ainsi devenir des adhérents au mouvement, ou des bénéficiaires de celui-ci. ${ }^{43}$

Les publics témoins sont ces segments du public que les mouvements sociaux cherchent à mobiliser pour leur cause. Ce sont des individus disposant d'une certain niveau de conscience sociale et politique, et qui, bien qu'ils ne soient généralement pas engagés, peuvent émerger en tant qu'observateurs et commentateurs en cas de rupture, mais aussi de restauration, de l'ordre public. ${ }^{44}$ Leurs réactions peuvent permettre la politisation rapide d'une question ou d'un événement, voire même influencer le fonctionnement des organes en charge de l'application des lois. ${ }^{45}$ Les publics témoins sont aussi nécessaires pour remodeler les normes sociales dominantes. L'établissement de nouvelles normes requiert une masse critique d'appui. Une fois qu'un point de basculement est atteint, l'institutionnalisation par le biais d'une codification légale devient possible. ${ }^{46}$ La masse critique se définit comme une « façon métaphorique souple de se référer à l'idée qu'un certain seuil de participants ou d'actions doit être dépassé avant qu'un mouvement social ne prenne vie $»^{47}$. La littérature concernant la masse critique examine rarement la question des origines communautaires des nouvelles normes. Cependant, nous pouvons tout de même noter le rôle significatif des publics témoins dans la facilitation de l'émergence de nouvelles normes socio-politiques et culturelles, et en tant que partie intégrante de la masse critique d'appui qui peut en résulter. Les réactions des témoins sont façonnées de façon dialectique par le militantisme social et les pratiques politico-légales, et inversement.

\section{HarassMap : origines et mission}

À la fin du mois d'octobre 2010, trois mois seulement avant la révolution, HarassMap a débuté ses opérations en lançant sa plateforme de crowd-mapping ${ }^{48}$ en ligne, hébergée par le site Ushashidi. Trois des cofondateurs de HarassMap avaient précédemment travaillé pour le CEDF, et avaient notamment lancé et géré la campagne "Rendre nos rues sûres pour tout le monde ». Le point fort du CEDF était l'usage de stratégies répandues au sein des $\mathrm{ONG}$ de sensibilisation et de plaidoyer à l'époque, à l'image du lobbying, de l'organisation d'ateliers de formation, d'activités de recherche, de l'écriture de rapports et de la fourniture de services juridiques pour des femmes dans le besoin. Au départ, la campagne "Rendre nos rues sûres pour tout le monde » différa du travail habituel du CEDF, en s'appuyant sur des bénévoles et en utilisant des techniques communautaires visant à obtenir l'appui du public, éléments perçus par les responsables de la campagne comme manquant au travail de la société civile en Égypte. ${ }^{49} \mathrm{Au}$ fur et à mesure du développement du programme, de l'augmentation de son financement et de l'embauche de personnel professionnel, les activités organisées sont redevenues plus conformes au travail de sensibilisation traditionnel commun aux ONG.

Les cofondateurs de HarassMap ont repris cette orientation vers la communauté dans le cadre de leur nouvelle initiative. L'élément sociétal était en effet essentiel au militantisme que les cofondateurs souhaitaient alors mettre en œuvre. Une grande partie de l'attention reçue par HarassMap de la part des médias occidentaux et des acteurs du développement international a été consacrée au crowd-mapping, applaudi et 
salué en tant qu'approche unique permettant de rendre visible le phénomène du harcèlement quotidien. Cependant, selon Rebecca Chiao, cofondatrice et directrice exécutive de HarassMap, l'élément technologique n'était envisagé que comme un «bonus » en plus du travail de terrain qu'ils voulaient effectuer dans les voisinages et les quartiers afin de transformer les mentalités de la société concernant le harcèlement. ${ }^{50}$ Des activités de sensibilisation politique et légale sur la question de la violence sexiste, dont le problème croissant du harcèlement sexuel, étaient déjà conduites par plusieurs ONG, à l'image du Centre El-Nadim et de son travail pionnier sur la torture et la violence sexuelle et domestique. A la fin de l'année 2008, 16 ONG ont formé le Groupe de travail pour l'interdiction de la violence sexuelle, coordonné par l'organisation New Woman Foundation. L'une de ses missions consistait à rédiger et promouvoir des amendements au code pénal relatifs à la violence sexiste.$^{51}$ Dans ce contexte, se concentrer sur la communauté et les perceptions sociales était pour les fondateurs de HarassMap une étape indispensable afin de transformer le climat sociopolitique général.

Pour les militants de HarassMap, la raison fondamentale pour laquelle le harcèlement sexuel existe est la tolérance sociale massive le concernant. Ils estiment que ce sont en fin de compte les gens qui permettent le harcèlement en ne s'exprimant pas et en n'intervenant pas en faveur de la victime lorsqu'ils sont témoins d'un incident. Leur mission première vise donc à remodeler les convictions et les comportements des témoins afin d'encourager les gens à percevoir le harcèlement comme un crime, à s'exprimer contre et à soutenir et aider les victimes de harcèlement en public. Le fait de se concentrer ainsi sur les convictions et les comportements des témoins est soustendu par l'idée que les structures politiques et légales seules ne sont pas suffisantes pour transformer les normes sociétales qui font du harcèlement une pratique acceptable. L'application de la loi aux cas de harcèlement sexuel a été et reste perçue comme hautement problématique. L'étude du CEDF de 2008 (la première publiée sur le phénomène en Égypte) indique que $97 \%$ des Égyptiennes et $87 \%$ des femmes étrangères enquêtées n'ont pas signalé à la police le harcèlement dont elles ont été victimes..$^{52}$ Ceci a été confirmé à nouveau par l'étude de HarassMap de 2014, qui montre que seulement $2 \%$ des femmes de l'échantillon ont signalé à la police les cas de harcèlement qu'elles ont subis ${ }^{53}$. L'étude du CEDF mettait notamment en évidence le fait que la police avait tendance à se moquer des femmes déposant plainte, que les femmes ne pensaient pas que la police pouvait les aider, et que les femmes étrangères considéraient les officiers de police eux-mêmes comme des harceleurs. De même, l'étude de HarassMap soulignait une préférence commune pour des méthodes alternatives et anonymes de signalement plutôt que le recours à la police, qui ne prenait pas le problème au sérieux. Selon Chiao, l'application effective de la loi dépend du fait que la police croie ou non qu'un acte répréhensible a été commis. Elle affirme ici que la croyance en la répréhensibilité de certaines actions est issue de racines sociales, et que les officiers de police eux-mêmes ne sont pas différents des autres membres de la société. Ils ne connaissent pas toujours la loi, ou ne sont pas toujours convaincus de son bien-fondé. ${ }^{54}$ 


\section{Créer de nouvelles normes sociales}

17 Une partie de l'approche de HarassMap visant à encourager les témoins à s'exprimer implique la construction et la promotion de nouvelles normes concernant le harcèlement sexuel. Ceci inclut :

Reconceptualiser le harcèlement sexuel, taharrush, afin que les actes non-physiques tels que les commentaires ou les regards, soient également perçus comme des formes de violence et de violation de l'espace personnel propres aux femmes. Autrement dit, il s'agit de s'éloigner du concept apparenté de $m u$ 'âksa (drague, séduction) ;

Affaiblir les stéréotypes répandus inhérents aux logiques de minimisation ou de justification du harcèlement sexuel, et interagir avec les gens pour leur permettre de percevoir la violence sexuelle différemment ;

Remettre en cause la stigmatisation des victimes, par le biais d'une rhétorique positive mettant en avant la force de celles qui subissent des cas de harcèlement, et s'opposer au système de genre binaire qui désavantage les femmes et limite leur participation publique ;

Promouvoir la perception et la définition du harcèlement sexuel quotidien comme un crime ayant des répercussions légales et sociales, et en particulier, inciter à de nouvelles formes de pression sociale pour prévenir le harcèlement sexuel ;

Exhorter les individus à s'exprimer et à intervenir contre le harcèlement, qu'ils en soient victimes ou témoins, ce qui peut impliquer l'encouragement à porter plainte, le développement de politiques relatives au harcèlement sexuel au sein des organisations, et la promotion de l'intervention et de l'expression contre le harcèlement comme des actes « cools ».

Une partie du travail de promotion de nouvelles normes consiste en campagnes d'information approfondies fréquemment déployées par HarassMap sur les réseaux sociaux, notamment Facebook et Twitter, ainsi que Tumblr dans une moindre mesure. Depuis 2012, HarassMap s'est lancé dans plusieurs grandes campagnes, dont «byitharrach lih?» (Pourquoi harcèle-t-il ?, campagne que les militants de HarassMap désignent par «Briser les mythes »), "salahha fi dimâghak » (Règle le problème dans ta tête), "mesh sâkta» (Je ne me tais pas), ainsi qu'une campagne plus récente liée à la Semaine internationale de lutte contre le harcèlement de rue, intitulée «di mesh $m u^{\prime a ̂} k s a, d a$ taharruch » (Ce n'est pas de la drague, c'est du harcèlement). La campagne «byitharrash lih?» avançait que les raisons souvent données par les gens pour expliquer le harcèlement sexuel, dont la pauvreté, l'illettrisme, le retard dans l'âge du mariage, la frustration sexuelle, l'insécurité ou encore les vêtements portés par les femmes, leur corps et leur présence en public, ne sont en réalité que des mythes. A travers l'un des messages de la campagne, HarassMap contredisait par exemple directement une justification économique fréquemment donnée pour expliquer le harcèlement, demandant ainsi « si la cause du harcèlement sexuel est la pauvreté, alors pourquoi les chefs d'entreprise harcèlent-ils les femmes?». L'organisation a aussi cherché à saper l'idée apparentée selon laquelle l'absence de travail conduit à l'impossibilité pour les jeunes hommes de se marier, créant ainsi chez eux une frustration sexuelle refoulée qui cause le harcèlement sexuel, en demandant: "si la cause du harcèlement sexuel est le mariage tardif, alors pourquoi les pères de famille harcèlent-ils les femmes? ». 


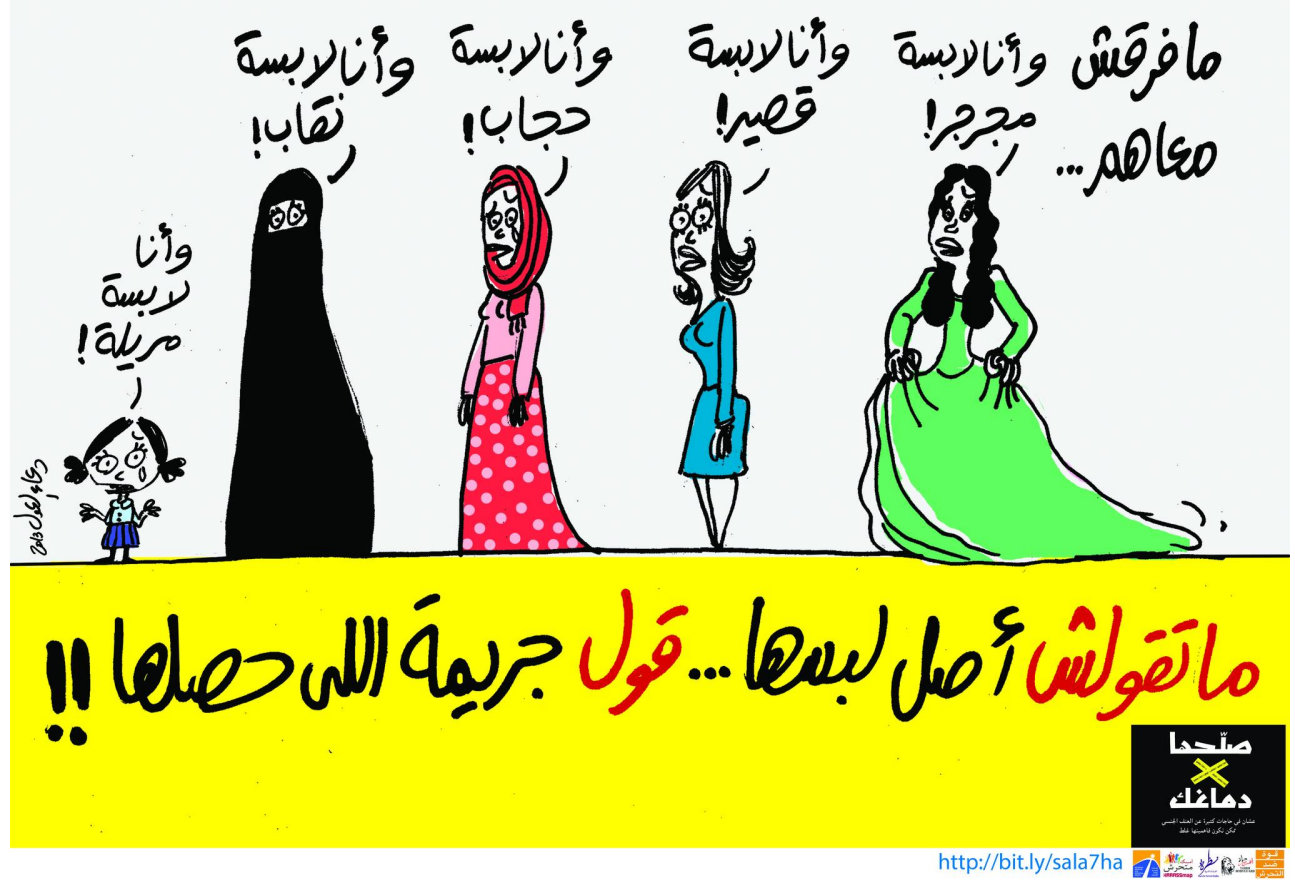

Cette image s'attaque à la tendance du public égyptien à faire un lien entre le style vestimentaire des femmes dans l'espace public, et le harcèlement sexuel ; elle illustre l'approche de HarassMap dans ses efforts pour influencer les normes sociales de rejet de la faute sur les victimes du harcèlement. L'image est extraite de la campagne "صلحها في دماغك" ("Corrige ça dans ta tête »), de nov.-déc. 2013, organisée dans le cadre de l'initiative « 16 jours d'activisme contre les violences sexistes » menée conjointement par HarassMap, Nazra for Feminist Studies, Egyptian Initiative for Personal Rights et Tahrir Bodyguard.

19 A la fin de l'année 2013, la campagne conjointe «salahha fi dimâghak», organisée avec Nazra pour les études féministes, l'Initiative égyptienne pour les droits personnels (Egyptian Initiative for Personal Rights) et Tahrir Bodyguards, visait à consolider différentes définitions de la violence sexuelle et à remettre en question l'inégalité entre les sexes. La campagne distinguait notamment les termes souvent confondus de taharrush ginsy (harcèlement sexuel), 'itidâ' ginsy (agression sexuelle) et ightișâb (viol). ${ }^{55}$ Un atelier organisé par HarassMap lors de la campagne, "la boîte des stéréotypes sexuels " (The Gender Box) avait pour objectif de sensibiliser les gens aux stéréotypes culturels relatifs à la masculinité et la féminité, et à la violence dont hommes et femmes peuvent faire l'objet en cas de non-conformité. La campagne «mesh sâkta» était la première à cibler les femmes. Composée d'une série d'affiches, de photos et de vidéos contenant des commentaires positifs et insistant sur le fait de s'exprimer contre le harcèlement, la campagne donnait des indications et des conseils sur la manière de faire face au harcèlement, par le biais d'une méthode collective permettant aux personnes intéressés de partager publiquement leur idées avec d'autres. Enfin, la campagne la plus récente, "di mesh mu'âksa, da taharruch» poursuit le travail de définition du taharruch en tant que harcèlement sexuel de rue quotidien, y compris verbal, et de distinction entre celui-ci et le flirt consenti et consensuel. Dans ce cadre, les messages de la campagne mettent en évidence les types de commentaire que les femmes entendent, en langue usuelle quotidienne, et qui relèvent du harcèlement sexuel, tel que « makana », littéralement traduit par « machine » et signifiant « salope ». 
Le but de la campagne est d'amener toute personne, victime ou témoin, entendant de tels commentaires, à s'exprimer.

Je ne garde pas le silence

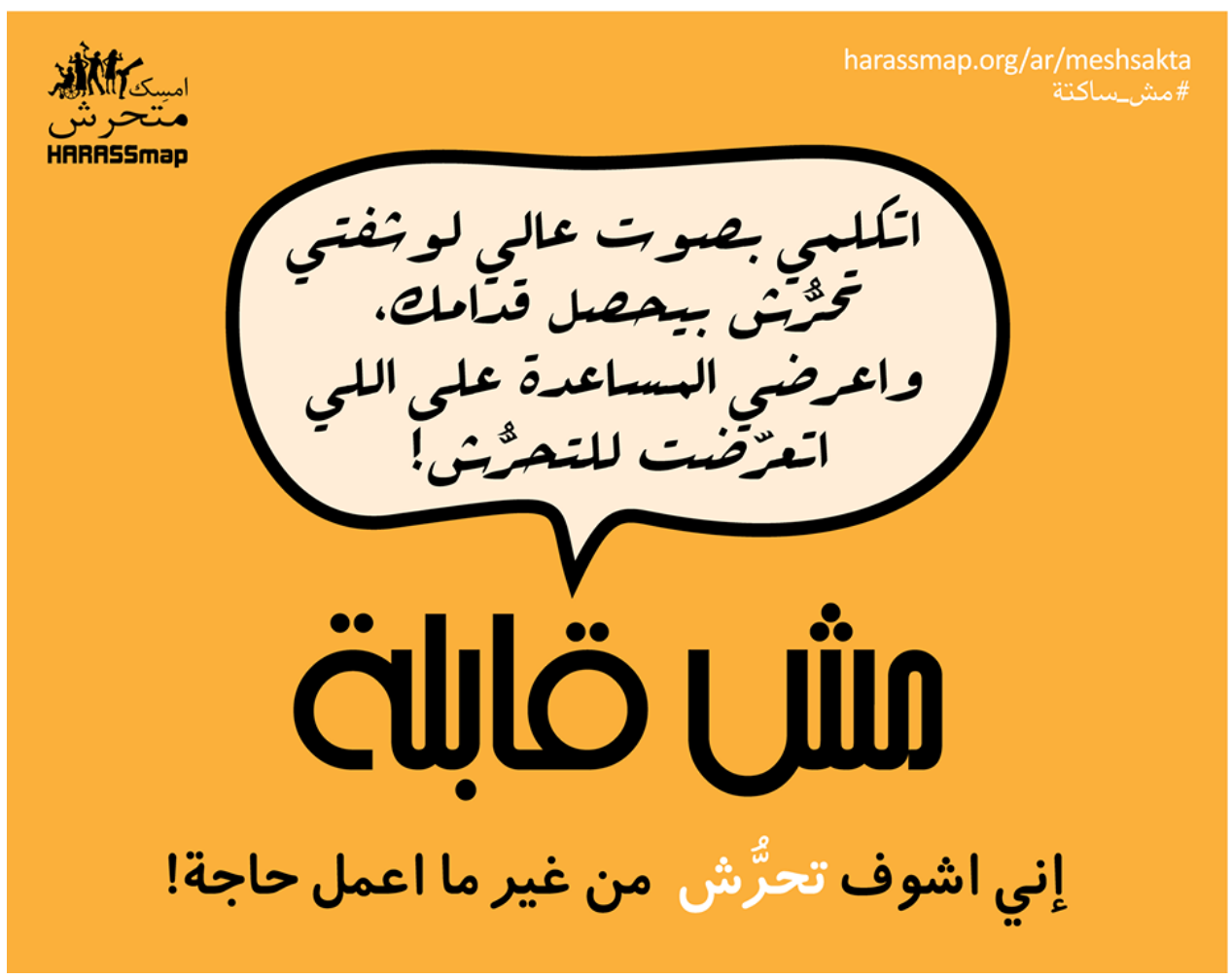

Cette image provient de la campagne de HarassMap "مش ساكتة" (" Je ne garde pas le silence »), du printemps 2014, qui ciblait spécifiquement les femmes victimes de harcèlement. Cependant, l'objectif ne se limitait pas à favoriser l'auto-affirmation de ces dernières; il s'agissait aussi de donner des conseils au public sur comment intervenir pour assister les personnes harcelées. Cette image reflète la mission fondamentale de HarassMap, qui est de contribuer à mettre fin à l'acceptabilité du harcèlement sexuel en demandant au public témoin/spectateur de prendre la parole.

En outre, faire percevoir le harcèlement sexuel quotidien aux témoins comme un crime est important en ce qu'il permet de les encourager à s'exprimer contre le problème en général. En particulier, opposer les réactions actives des témoins dans des cas de vol à l'absence de réactions au harcèlement sexuel, est un procédé très répandu. Au travers de ses messages de campagne, HarassMap associe violations physiques et vols de biens matériels. Sur l'une des affiches de la campagne "salahha fi dimâghak», HarassMap et ses partenaires avaient par exemple écrit: "Celui qui vole ton portefeuille est un criminel » et « Celui qui vole ton corps et viole ton sourire est un criminel ».

\section{L'intervention des témoins et la création d'une masse critique}

En dépit des peurs selon lesquelles le travail de lutte contre le harcèlement sexuel engendrerait des hommes indisciplinés nécessitant d'être contrôlés ou de se réformer, les cibles des interventions de HarassMap ne sont généralement pas les harceleurs ou les femmes victimes de harcèlement sexuel. Afin de mettre un terme à l'acceptabilité sociale du harcèlement sexuel, la position philosophique de HarassMap est que tous les membres de la société doivent prendre la responsabilité personnelle de s'exprimer contre cette pratique, en particulier ceux qui se tiennent à l'écart et ignorent ou 
assistent à des cas de harcèlement sexuel sans rien faire. Pour HarassMap, parvenir à convaincre les gens que le harcèlement sexuel est un crime et à leur faire surmonter leurs peurs ou leurs préjugés représenterait un changement de norme crucial qui les rendrait disposés à prendre position, à s'exprimer contre les harceleurs et à offrir leur soutien aux victimes de harcèlement.

Le but premier de l'Unité d'intervention communautaire de HarassMap est de faciliter l'établissement d'une éthique disciplinaire et d'un sens de la responsabilité morale afin d'encourager les témoins à intervenir, à aider les membres de leur communauté dans le besoin et à ne pas tolérer de comportements criminels dans leur quartier. Deux fois par an, HarassMap forme une brigade de "capitaines » qui dirigent des équipes locales de bénévoles dans leurs quartiers afin de mener des campagnes de sensibilisation sur le terrain, dans les rues. Des formations mobiles mensuelles de tous les bénévoles sont aussi organisées dans tous les gouvernorats d'Égypte. Les bénévoles sont recrutés par le biais des réseaux sociaux, et les équipes de bénévoles utilisent également les réseaux sociaux lorsqu'elles mettent en œuvre leurs propres efforts de sensibilisation des communautés, en postant des photos et des messages depuis la rue. Au travers de ces efforts de sensibilisation de la communauté, les témoins se transforment en adhérents au mouvement et encouragent d'autres témoins à faire de même. Au minimum, les bénévoles cherchent à convaincre les témoins d'exprimer leur désaccord ou leur indignation lorsque les valeurs sociales relatives à l'intégrité physique des femmes font publiquement l'objet de violations. Les capitaines et les bénévoles sont formés sur les questions d'inégalité entre les sexes, de violence. Ils apprennent aussi des réponses efficaces aux stéréotypes sexistes souvent avancés par les membres de la communauté. Les bénévoles sont de plus formés à faire débattre les gens sur les normes relatives au harcèlement sexuel, tout en évitant l'agressivité et l'ergoterie et en empêchant les conversations de diverger vers la stigmatisation des victimes, ainsi qu'à conclure les conversations en obtenant la confirmation des témoins qu'ils ne resteront plus silencieux et s'exprimeront lorsqu'ils verront un cas de harcèlement se produire.

Deux nouveaux programmes apparentés, Écoles et Universités Sûres (EUS) et Zones sûres $(\mathrm{ZS})^{56}$, ont encore élargi le champ de la lutte contre l'effet du témoin à plus grande échelle. Les programmes EUS et ZS cherchent à sensibiliser les individus dès leur plus jeune âge aux questions d'intégrité physique et de comportements constitutifs $\mathrm{du}$ harcèlement, ainsi qu'à encourager les entreprises et autres entités publiques à développer des politiques de tolérance zéro et des procédures officielles contre le harcèlement sexuel. La stratégie du programme Zone sûres prévoit un travail avec les petits commerces, en procédant rue par rue afin de construire des réseaux de soutien parmi les propriétaires de commerces, à la fois afin d'empêcher les réactions violentes des harceleurs et de servir de modèle positif à suivre pour que d'autres commerces établissent des politiques anti-harcèlement sexuel. Une campagne d'information récente liée au programme EUS, "'âyzîn siyâsa gowa al-gâm'a» (Nous voulons une politique à l'université), a appelé les universités égyptiennes à développer de telles politiques. ${ }^{57}$

Il est important de noter que la plateforme de crowd-mapping de HarassMap, qui a retenu l'attention de la communauté internationale, constitue à la fois un espace et un outil essentiels pour transformer les perceptions sociales. Plateforme interactive en ligne qui allie SIG et SMS, elle fournit aux gens la possibilité de signaler anonymement des cas de harcèlement et de cartographier leurs témoignages en ligne ou par le biais de 
SMS. Ceux-ci deviennent ensuite visibles de façon publique via Google Maps sur le site internet de HarassMap. Skalli mentionne que cet outil en ligne a pour objectif de fournir un espace sûr permettant aux femmes de raconter leur histoire sans honte et sans représailles..$^{58}$ L'idée de sécurité est importante étant donné le manque de soutien des organismes en charge de l'application de la loi à celles qui tentent de porter plainte par le biais des voies officielles. Dans ces circonstances, Young précise que la carte sert d'outil de documentation alternatif, "permettant aux victimes de contourner les contraintes institutionnelles [...] qui peuvent les empêcher de signaler un incident ${ }^{59}$. La carte donne aussi aux individus la possibilité de récuser directement les discours de stigmatisation des victimes, et de s'exprimer à propos de leurs peurs, leurs frustrations et leur colère contre leur harceleur, contre la société qui normalise la pratique du harcèlement sexuel, et contre le manque de recours légaux efficaces.

Une critique récente de l'approche du témoin défend l'idée qu'elle ne résout pas le problème sous-jacent de la violence sexuelle. Elk et Devereaux notent que le fait de demander aux témoins d'intervenir en cas de violence sexuelle, comme le viol, crée pour eux un danger inhérent et que les témoins, comme les victimes, ne reçoivent pas le soutien approprié..$^{60}$ Ils indiquent que cela ne fait que modifier la cible des discours de stigmatisation des victimes, dont les témoins qui échouent à fournir une assistance en cas de besoin se mettent également à faire l'objet. Selon eux, l'approche du témoin semble n'être rien de plus qu'une autre forme de vigilantisme, qu'ils affirment être une caractéristique de l'État " carcéral », élaborée dans le but de punir et non de réhabiliter les délinquants. "Même lorsque l'intervention d'un témoin est réussie, interrompre une agression n'est pas la même chose que mettre un terme à la violence. Il ne s'agit même pas de prévention de la violence » (Elk \& Devereaux 2014). Enfin, ils soutiennent que l'approche du témoin n'oblige aucunement les individus à l'introspection et à s'intéresser à la violence qu'ils sont eux-mêmes capables de commettre.

De tels arguments éludent la complexité qui entoure les campagnes d'information des mouvements sociaux et la question du comportement des témoins. Ils n'expliquent en quoi consiste l'approche du témoin. L'impression dominante est que l'intervention physique est requise, mais d'autres formes d'interventions, tout aussi utiles et salutaires, et ne conduisant pas les témoins à se mettre en danger, ne sont pas évoquées. De plus, les témoins en Égypte ne sont pas nécessairement opposés à l'intervention physique s'ils considèrent qu'elle est justifiée par la situation. C'est le cas avec le vol, au sujet duquel il est connu et fréquent que des voisinages entiers se rassemblent pour attraper un voleur, de jeunes hommes formant souvent des factions ad hoc et se munissant de bâtons, de barres de fers et de chaines pour ce faire. ${ }^{61}$

La rhétorique de HarassMap sur l'intervention des témoins accepte de façon implicite que parmi le groupe de témoins, il pourra y avoir des harceleurs. Pour éviter une rhétorique accusatrice qui blâmerait un groupe particulier de gens pour le harcèlement sexuel, leur discours est plutôt centré sur le besoin de reconnaître que chaque individu a une responsabilité envers les autres membres de sa communauté. Plutôt que de déplacer le blâme, ce message remodèle subtilement la nature de la responsabilité sociale en demandant aux gens de reconsidérer leurs propres biais les poussant à garder le silence ou les empêchant de voir l'humanité fondamentale des victimes de violence sexuelle. Voir l'approche du témoin comme une pratique qui déplace la stigmatisation de la victime et qui ignore la question du potentiel violent des individus est problématique, en ce que cela repose sur une approche binaire particulière 
d'inspiration occidentale qui oppose l'individu et la communauté. La notion de responsabilité individuelle promeut seulement le besoin pour les gens de se contrôler et les dissocie de toute responsabilité envers la communauté à laquelle ils appartiennent. Pour cette raison, promouvoir la responsabilité communautaire est souvent perçu comme inefficace ainsi que comme une forme d'hégémonie.

Construire une masse critique de publics témoins représente le but ultime de HarassMap afin de mettre un terme à l'acceptabilité sociale du harcèlement sexuel en Égypte. Au cœur de cela, les efforts entrepris pour parvenir au changement social supposent la construction de soutiens sur le terrain afin de que de nouvelles normes et comportements introduits par les initiatives prennent racine, individu par individu, et deviennent finalement ordinaires au sein de l'opinion publique. Une fois que cela se produit et que suffisamment d'individus sont convaincus de ou mettent en pratique les nouvelles normes promues par HarassMap et d'autres initiatives similaires, un point de basculement est alors atteint. Ici, lorsque suffisamment de gens seront devenus insatisfaits des pratiques sociales, politiques ou légales ne correspondant pas aux nouvelles normes, ceux-ci se mettront alors à réclamer un changement. Cette masse critique est perçue comme essentielle pour amener un changement politique et légal puisque les militants de HarassMap affirment que le gouvernement n'a aucune incitation au changement, malgré le travail important des entités officielles de la société civile. Une masse critique soutenant de nouvelles pratiques normatives a ici la possibilité de menacer la légitimité du gouvernement. Il est supposé premièrement que l'État adhérera à la volonté sociale du peuple et intégrera les nouvelles normes à des instruments politiques et légaux, et deuxièmement que les représentants de l'État sont eux-mêmes des membres de la société, et adopteront ces nouvelles normes dans leur propre système de valeurs.

\section{Conclusion}

Cet article a défendu le besoin de repenser le politique et la dépolitisation en ce qui concerne les interventions sociales et culturelles du militantisme anti-harcèlement sexuel en Égypte. De telles interventions ne plaident pas directement pour le changement auprès de l'État mais constituent bien des processus politiques cherchant à transformer la nature des convictions et des pratiques des gens, ainsi que l'engagement de l'État contre le problème du harcèlement sexuel. La relation entre harcèlement sexuel politiquement motivé et socialement motivé, en terme de facteurs causaux et de normes communes rendant le harcèlement sexuel socialement acceptable et renforçant les pratiques patriarcales, souligne l'intégration des sphères politique et sociale. Étant donné cette intégration, les interventions centrées sur le remodelage des normes sociales et culturelles ont un impact indéniable sur la pratique politique. L'approche de HarassMap en matière de changement des normes sociales, d'encouragement des témoins à s'exprimer et de création d'une masse critique, ne dépolitise pas le problème du harcèlement sexuel, au sens où le but ultime de leur militantisme est de forcer l'État à garantir un environnement dans lequel les citoyens se sentent protégés de la violence publique. Les militants de HarassMap tentent de parvenir à ce résultat par le biais d'une approche de terrain qui remodèle les normes sous-tendant le système sociopolitique actuel au sein duquel le harcèlement sexuel est toléré, n'est pas considéré comme un crime, et pour lequel personne n'est tenu de rendre des comptes (pas même l'État). Le 
changement politique et une réaction plus impliquée de l'État sont ici une suite du militantisme de HarassMap à l'échelle de la communauté : ils se produisent lorsque le public lui-même s'exprime et réclame de l'État qu'il adopte des lois équitables et qu'il les applique activement, afin que l'espace public devienne libre de toute violence sexuelle.

\section{BIBLIOGRAPHIE}

ABU LUGHOD, Lila. 2013. Do Muslim Women Need Saving? Cambridge: Harvard University Press.

ABU LUGHOD, Lila. 2011. The Active Social Life of "Muslim Women's Rights": A Plea for Ethnography and Polemic, With Cases from Egypt and Palestine. Journal of Middle East Women's Studies, 6(1): 1-45. ABDELRAHMAn, Maha. 2004. Civil Society Exposed: The Politics of NGOs in Egypt. Cairo: The American University in Cairo Press.

AHMAD, Tania. 2014. Socialities of Indignation: Denouncing Party Politics in Karachi. Cultural Anthropology, 29(2): 411-432.

AHMAD-ZAKI, Hind \& ABD ALHAMID, Dalia. 2014. Women as Fair Game in the Public Sphere: A Critical Introduction for Understanding Sexual Violence and Methods of Resistance. Jadaliyya, July 9 , 2014. http://www.jadaliyya.com/pages/index/18455/women-as-fair-game-in-the-publicsphere_a-critical.

ALVAREZ, Sonia E., DAGNINO, Evelina, and ESCOBAR, Arturo. 1998. Introduction: The Cultural and the Political in Latin American Social Movements. In Cultures of Politics and Politics of Cultures: ReVisioning Latin American Social Movements, (eds.) Sonia Alvarez, Evelina Dagnino, et Arturo Escobar. Boulder: Westview Press.

AMAR, Paul. 2011. Turning the Gendered Politics of the Security State Inside Out? International Feminist Journal of Politics, 13(3): 299-328.

BIANCHI, Robert. 1989. Unruly Corporatism: Associational Life in Twentieth Century Egypt. Oxford: Oxford University Press.

BRAUN, Robert \& KOOPMANS, Paul. 2014. Watch the Crowd: Bystander Responses, Trickle Down Politics, and Xenophobic Mobilization. Comparative Political Studies, 47(4) : 631-658.

BRAUN, Robert \& KOOPMANS, Paul. 2012. "Bystander Responses and Xenophobic Mobilization," Document de réflexion, Wissenschaftszentrum Berlin fur Sozialforschung. http:// www.econstor.eu/handle/10419/68460.

CHEKRoun, Peggy \& BRAUER, Markus. 2002. The Bystander Effect and Social Control Behavior: The Effect of the Presence of Others on People's Reactions to Norm Violations. European Journal of Social Psychology, 32: 853-867.

DARLEY, J.M. \& LATANE, B. 1968. Bystander Intervention in Emergencies: Diffusion of Responsibility. Journal of Personality and Social Psychology, 8: 377-383. 
EGYPTIAN CENTER FOR WOMEN'S RIGHTS. 2009. Sexual Harassment in the Arab Region: Cultural Gaps and Legal Challenges. ECWR Report, (ed.) Nehad Abul Komsan. Print.

EL-DEEB, Bouthaina. 2013. Study on Ways and Methods to Eliminate Sexual Harassment in Egypt. UN Women, http://harassmap.org/en/wp-content/uploads/ 2014/02/287_Summaryreport_eng_low-1.pdf.

ELK, Lauren Chief \& DEVEREAUX, Shaadi. 2014. “The Failure of Bystander Intervention.” New Inquiry, 12-23-2014. Consulté le 01-04-2015. http://thenewinquiry.com/essays/failure-ofbystander-intervention/.

ELSAYED, Heba \& RIZzo, Helen. 2014. Media, Political Opportunity and the Anti-Sexual Harassment Campaign in the Post-2011 Egypt. Manuscrit non publié.

FAHMY, Amel, ABDELMONEM, Angie, HAMDY, Enas, BADR, Ahmed \& HASSAN, Rasha. 2014. “Toward a Safer City: Sexual Harassment in Greater Cairo: Effectiveness of Crowdsourced Data." Rapport de HarassMap (rapport complet), http://harassmap.org/en/wp-content/uploads/2013/03/TowardsA-Safer-City_full-report.pdf.

FEREE, Myra Marx. 2003. Resonance and Radicalism: Feminist Framing in the Abortion Debates of the United States and Germany. American Journal of Sociology, 109(2): 304-344.

FIDH, Nazra for Feminist Studies, New Women Foundation and Uprising of Women in the Arab World. 2014. "Egypt Keeping Women Out: Sexual Violence Against Women in the Public Space." http://www.fidh.org/IMG/pdf/egypt_women_final_english.pdf.

FINNEMORE, Martha. 1996. Norms, Culture, and World Politics: Insights from Sociology's Institutionalism. International Organization, 50(2): 325-347.

FINNEMORE, Martha \& SIKKINK, Kathryn. 1998. International Norm Dynamics and Political Change. International Organization, 52(4): 887-917.

HAFEZ, Sherine. 2014. The Revolution Shall Not Pass Through Women's Bodies: Egypt, Uprising and Gender Politics. The Journal of North African Studies, 19(2): 172-185.

HASSAN, Rasha, SHOUKRY, Aliyaa \& ABUL KOMSAN, Nehad. 2008. “Clouds in Egypt's Sky: Sexual Harassment: From Verbal Harassment to Rape.” ECWR Report, http://egypt.unfpa.org/Images/ Publication/2010_03/6eeeb05a-3040-42d2-9e1c-2bd2e1ac8cac.pdf.

LANGOHR, Vickie. 2014. New President, Old Pattern of Sexual Violence in Egypt. Middle East Report, July 7. http://www.merip.org/mero/mero070714.

LANGOHR, Vickie. 2013. "This is Our Square”: Fighting Sexual Assault at Cairo Protests. Middle East Report, 238 (Fall): 18-35.

LEVITT, Peggy \& Merry, Sally. 2009. Vernacularization on the Ground: Local Uses of Global Women's Right in Peru, China, India and the United States. Global Networks, 9(4): 441-461.

MCCARTHY, John D. \& ZALD, Mayer N. 1977. Resource Mobilization and Social Movements: A Partial Theory. American Journal of Sociology, 82(6): 1212-1241.

MERRY, Sally Engle. 2006. Human Rights and Gender Violence: Translating International Law into Local Justice. Chicago: University of Chicago Press.

MICHAEL, Maggie. 2013. "Mob Kills Alleged Thief in Egypt Vigilante Attack.” AP, Yahoo News, 3-21-2013. Consulté le 01-04-2015. http://news.yahoo.com/mob-kills-alleged-thief-egyptvigilante-attack-200620533.html. 
Nazra for Feminist Studies. 2014. Concept Paper: Different Practices of Sexual Violence Against Women (English). http://nazra.org/en/2014/02/concept-paper-different-practices-sexualviolence-against-women.

RISSE, Thomas \& SIKKINK, Kathryn. 1999. The Socialization of International Human Rights Norms into Domestic Practices. In The Power of Human Rights: International Norms and Domestic Change, Thomas Risse, Stephen C. Ropp et Kathryn Sikkink (eds). Cambridge: Cambridge University Press. RIZZO, Helen, PRICE, Anne M., \& MEYER, Katherine. 2012. Anti-Sexual Harassment Campaign in Egypt. Mobilization: An International Journal, 17(4): 457-475.

RIZZo, Helen, PRICE, Anne M., \& MEYER, Katherine. 2008. “Targeting Cultural Change in Repressive Environments: The Campaign Against Sexual Harassment in Egypt." Rapport du CEDF http:// ecwronline.org/pdf/studies/AntiHarassment_for_ECWR.pdf.

ROGERS, Everett M. 1983. The Diffusion of Innovation. New York: The Free Press.

RUBIN, Jeffrey W. 1996. "Decentering the Regime: Culture and Regional Politics in Mexico." Latin American Research Review, 31(3): 85-126.

SCOTT, Robet. 2000. The Limits of Behavioral Theories of Law and Social Norms. Virginia Law Review, 86(8): 1603-1647.

SKALLI, Loubna Hanna. 2014. Young Women and Social Media Against Sexual Harassment. JOURNAL of North African Studies, 19(2): 244-258.

SNOW, David A., ZURCHER , Louis A., \& Peters, Robert. 1981. Victory Celebrations as a Theater: A Dramaturgical Approach to Crowd Behavior. Symbolic Interaction, 4(1): 21-42.

SWIDLER, Ann. 1995. "Cultural Power and Social Movements." In Social Movements and Culture, (eds.) Hank Johnston and Bert Klandermans. Minneapolis: University of Minnesota Press.

TADROS, Mariz. 2014. Reclaiming the Streets for Women's Dignity: Effective Initiatives in the Struggle Against Gender-Based Violence in Between Egypt's Two Revolutions.

TADROS, Mariz. 2013a. "Whose Shame Is It? The Politics of Sexual Assault in Morsi’s Egypt." Heinrich Boll Stiftung, Afrique Du Nord Tunis, http://tn.boell.org/downloads/MarizTadros.pdf. TADROS, Mariz. 2013b. Politically Motivated Sexual Assault and the Law in Violent Transitions: A Case Study From Egypt. Institute of Development Studies, http://opendocs.ids.ac.uk/opendocs/ bitstream/handle/123456789/2950/ER8\%20final\%20online.pdf? sequence $=1 \& u t m \_s o u r c e=i d s w e b s i t e \& u t m \_$medium=download\&utm_campaign=opendocs. THORNBERG, Robert. 2007. A Classmate in Distress: School Children as Bystanders and Their Reasons for How They Act. Social Psychology of Education, 10: 5-28.

Wikipedia. Critical Mass (Sociodynamics) entry, last accessed August 5, 2014. http:// en.wikipedia.org/wiki/Critical_mass_(sociodynamics).

YoUNG, Chelsea. 2014. HarassMap: Using Crowdsourcing Data to Map Sexual Harassment in Egypt. Technology Innovation Management Review, http://timreview.ca/sites/default/files/ article_PDF/Young_TIMReview_March2014.pdf.

ZWINGEL, Susanne. 2012. How Do Norms Travel: Theorizing International Women's Rights in Transnational Perspective. International Studies Quarterly, 56 : 115-129. 


\section{NOTES}

1. Hassan et al. 2008.

2. NDT : axé sur la communauté ou communautaire, community-based en anglais, est utilisé dans cet article pour décrire une activité qui est organisée et se déroule au niveau local, qui prend la communauté, par opposition aux institutions et à l'État notamment, pour cible et pour agent de changement à la fois.

3. ECWR 2009; Rizzo et al. 2008 \& 2012.

4. Tadros 2013a. L'argument de Tadros ne mentionne pas directement le CEDF, mais les interventions sociales de façon plus générale. Son analyse examine la manière dont les sentiments politiques personnels influencent les perceptions liées au harcèlement sexuel et soulève le problème de l'absence de limites claires entre harcèlement politiquement motivé et formes quotidiennes de harcèlement sexuel, qui crée une forme de confusion permettant la reproduction $\mathrm{du}$ harcèlement politiquement motivé.

5. Abu Lughod 2011, p. 14 \& 2013, p. 156.

6. NDT : Agence des Nations-Unies pour l'égalité des sexes et l'autonomisation des femmes.

7. El-Deeb 2013.

8. ElSayed \& Rizzo 2014, manuscrit non publié.

9. Ahmad 2014, p.427. L'analyse d'Ahmad est centrée sur les réactions publiques aux manifestations de 2007 qui ont conduit à un conflit violent entre le gouvernement et l'opposition à Karachi au Pakistan. Elle défend l'idée que, plutôt que de prendre part aux manifestations et au conflit, une partie de la population de Karachi a volontairement choisi de rester enfermée chez elle. Selon elle, cet enfermement représente une forme d'engagement politique.

10. Alvarez et al. 1998; Rubin 1996; Swidler 1995.

11. Rubin 1996, p. 89, citant Foucault 1990, pp. 93-96.

12. Tadros 2013a\&b \& 2014.

13. Hassan et al. 2008; El Deeb 2013; Fahmy et al. 2014.

14. Langohr 2013 \& 2014; Tadros 2013b; Ahmed-Zaki \& Abd Alhamid 2014.

15. Amar 2011.

16. Rizzo et al. $2012 b$.

17. Ibid.

18. Ibid, p. 467.

19. Abu Lughod 2010, p. 14.

20. Amar 2011, p. 314-316.

21. Abdelrahman 2004; Bianchi 1989.

22. Elsayed \& Rizzo 2014, manuscrit non-publié.

23. Langohr 2013; Hafez 2014.

24. Interview 2014, Amal ElMohandes de l'ONG Nazra for Feminist Studies; conversations personnelles de 2014 avec Hussein el-Shafei et Noora Flinkman de HarassMap.

25. Langohr 2014; Interview 2014, Farah Shash de l'organisation El-Nadim.

26. Interview de juin 2014.

27. Tadros 2013a, p. 6.

28. Skalli 2014.

29. Ilahi 2008; Peoples 2008; Rizzo et al. 2012a\&b.

30. Tadros 2014, p. 10.

31. Finnemore 1996, p 891.

32. Zwingel 2012.

33. NDT : les entrepreneurs de normes (norm entrepreneurs) regroupent tous les acteurs quels qu'ils soient - individus, entreprises, ONG, États, organisations multilatérales - qui promeuvent délibérément et activement la diffusion d'une ou de plusieurs normes.

Égypte/Monde arabe, 13 | 2015 
34. Feree 2003; Levitt \& Merry 2009.

35. Alvarez et al. 1998, p. 7.

36. Community Outreach Director.

37. Conversation personnelle, août 2014.

38. Chekroun \& Brauer 2002.

39. Darley \& Latane 1968; Thornberg 2007.

40. Braun \& Koopman 2012 \& 2014.

41. Banyard et al. 2004.

42. Braun \& Koopman 2012 \& 2014.

43. McCarthy \& Zald 1977; Snow et al. 1981.

44. Snow et al. 1981.

45. Ibid.

46. Finnemore \& Sikkink 1998.

47. Oliver et al. 1985.

48. NDT : le crowd-mapping consiste à cartographier et à centraliser numériquement des incidents ou des événements signalés par des témoins, ou bien des victimes.

49. Rizzo et al. 2012a\&b

50. Interview, Mars 2014

51. FIDH 2014

52. Hassan et al. 2008.

53. Fahmy et al. 2014

54. Interview, Mars 2012

55. Nazra 2014

56. Safe Schools and Universities et Safe Areas.

57. En 2014, l'Université du Caire a attiré l'attention des communautés nationale et internationale, du fait d'un cas de harcèlement contre une jeune femme ayant enlevé son abaya après être entrée à la faculté de droit, tout en restant entièrement couverte et voilée. A la suite de l'incident, des professeurs, en coopération avec des initiatives de lutte contre le harcèlement, ont élaboré une politique anti-harcèlement sexuel.

58. Skalli 2014, p. 251.

59. Young 2014, p. 6.

60. Elk \& Devereaux 2014.

61. Michael 2013. L'agence Associated Press (AP) a raconté l'histoire d'une ville du Delta où les habitants ont lynché un homme pour avoir volé une voiture. Cette histoire est un exemple extrême de la manière dont les membres de la communauté peuvent intervenir en cas de vol, bien que l'angle de l'article de AP sur le lynchage ait visé à souligner l'anarchie croissante de la période post-révolutionnaire. De telles réactions sont typiques des interventions en cas de vol.

\section{RÉSUMÉS}

Cet article explique en quoi le travail réalisé au niveau communautaire par les initiatives antiharcèlement sexuel représente un processus politique pour venir à bout du harcèlement en Egypte. Depuis la révolution égyptienne, les initiatives d'orientation communautaire ont employé des stratégies visant à transformer les perceptions sociales et les comportements liés au 
harcèlement sexuel dans l'espace public. Ces stratégies, comme celles de HarassMap, comprennent la conduite de campagnes de sensibilisation au niveau de la rue et l'emploi de plateformes technologiques pour recadrer la notion de responsabilité sociale et bousculer les stéréotypes de genre. Pourtant, nombre de chercheurs ont remis en question ce travailparticulièrement les activités plus anciennes du Egyptian Center for Women's Rights (ECWR)estimant qu'il représente un évitement de l'engagement politique et ne se confronte pas aux inégalités structurelles de genre. Cet article utilisera l'argument de Finnemore et Sikkink (1998) sur l'émergence des normes pour soutenir que les initiatives anti-harcèlement sexuel comme HarassMap envisagent le changement social à travers la construction d'une masse critique autour de nouvelles normes sociales. En minant les normes patriarcales rejetant la faute sur les victimes, et en encourageant le public à intervenir contre le harcèlement dans la sphère publique, HarassMap crée une nouvelle représentation de la responsabilité sociale. Quand un seuil critique est atteint, les activistes de HarassMap sont d'avis qu'une revendication publique émergera pour forcer des réformes étatiques, juridiques et politiques, pour protéger les femmes dans l'espace public. De telles approches axées sur le niveau communautaire sont politiques, mais les groupes réformistes comme HarassMap ne recherchent de tels résultats que lorsqu'il existera suffisamment de volonté publique de changement.

This paper argues that the community-based work of anti-sexual harassment initiatives represents a political process toward ending sexual harassment in Egypt. Since the Egyptian Revolution, community-based initiatives have employed strategies to transform social perceptions and behaviors regarding public sexual harassment. The strategies of these new initiatives, like HarassMap, include conducting street outreach campaigns and employing technological platforms to reframe the nature of social responsibility and to disrupt gendered stereotypes. Yet, a number of scholars have challenged this community-based anti-harassment work, particularly the early work of the Egyptian Center for Women's Rights (ECWR), for avoiding political engagement and failing to address structural gender inequalities. Drawing on Finnemore and Sikkink's (1998) argument regarding norm emergence, this paper maintains that anti-sexual harassment initiatives, like HarassMap, employ an approach to social change that aims to build a critical mass around new social norms. By undermining patriarchal norms that blame victims and encouraging people to intervene against public sexual harassment, HarassMap is creating a new vision of social responsibility. When a tipping point is reached, HarassMap activists believe that public outcry will then force political and legal reform from the state to protect women in public space. Such community-based approaches are political but the reform entities like HarassMap seek results only when there is enough public will for change.

\section{INDEX}

Mots-clés : Égypte, femmes, harcèlement sexuel, violence, résistance, harassmap

Keywords : Egypt, women, sexual harassment, violence, resistance, harassmap, bystander approach

\section{AUTEURS}

\section{ANGIE ABDELMONEM}

Angie Abdelmonem est actuellement doctorante en anthropologie à l'École de l'Evolution Humaine et du Changement Social (School of Human Evolution and Social Change) à l'Université d'État de l'Arizona. Sa spécialisation porte sur les ONG, les mouvements sociaux, le 
développement et la violence sexiste. Sa thèse examine le rôle des entités de la société civile et des initiatives indépendantes en matière de lutte contre le harcèlement sexuel de rue. En 2005-2006, elle a effectué un stage d'un an au sein du Centre Égyptien pour les Droits des Femmes (Egyptian Center for Women's Rights), travaillant sur des projets relatifs au harcèlement sexuel, aux mutilations génitales féminines et à l'obtention du statut consultatif auprès des Nations Unies. Entre 2013 et 2014, elle a passé l'année à effectuer un travail d'observation participante au sein de HarassMap, ainsi qu'à observer et interviewer des militants de plusieurs autres ONG et initiatives indépendantes luttant contre le harcèlement sexuel, dont Ḍed el-Taharrush, Harakat Bassma, Shoft Taharrush, and Nazra pour les études féministes (Nazra for Feminist Studies). Depuis 2015 et jusqu'à 2017, elle participe à un projet conjoint financé qui examine l'intersection entre médias de masse, violence sexiste publique et politiques de respectabilité. Publications: ABDELMONEM, Angie. 2015. "Reconceptualizing Sexual Harassment: A Longitudinal Assessment of El-Taharrush El-Ginsy in Arabic Online Forums and Anti-Sexual Harassment Activism." Kohl: Journal of Gender and Body Research. FAHMY, Amel, ABDELMONEM, Angie, HAMDY, Enas, BADR, Ahmed \& HASSAN, Rasha. 2014. "Toward a Safer City: Sexual Harassment in Greater Cairo: Effectiveness of Crowdsourced Data." HarassMap Report (rapport complet en anglais), http://harassmap.org/en/ wp-content/uploads/2013/03/Towards-A-Safer-City_full-report.pdf.

angie.abdelmonem@asu.edu 